\title{
高容量超级电容器电极材料的设计与制备
}

\author{
吴 中 ${ }^{1,2}$ 张新波 $^{1, *}$ \\ ('中国科学院长春应用化学研究所, 稀土国家重点实验室, 长春 $130022 ;{ }^{2}$ 中国科学院大学, 北京 100049)
}

\begin{abstract}
摘要: 超级电容器寿命长, 安全性高, 并可以实现快速充放电, 是化学电源研究的热点之一。然而, 超级电 容器的能量密度较低限制了其更多的应用。因此, 超级电容器领域的研究关注点在如何提高超级电容器的能 量密度。其中, 提高比容量是提高能量密度的一种有效途径。本文通过对电极材料和电解液的优化来研究制 备得到高容量超级电容器的方法。电极材料的比表面积、孔道结构和导电性对其电化学性能有着直接的影 响。一方面, 通过优化电极材料的孔道结构和比表面积可以增加活性位点并提高电解液离子传导率, 从而得 到高比电容。另一方面, 电极材料导电性的提高有利于提升其电子传导率从而得到较高的比容量。本文分别 对碳材料和金属氧化物/氢氧化物的优化达到了增加双电层电容和赝电容的目的。不仅如此, 还可以通过在电 解液中增加氧化还原电对从而得到高比电容。这一方法为高容量超级电容器的制备提供了新的思路。
\end{abstract}

关键词: 超级电容器; 比容量; 电极材料; 电解液; 双电层电容; 赝电容 中图分类号: 0646

\section{Design and Preparation of Electrode Materials for Supercapacitors with High Specific Capacitance}

\author{
WU Zhong ${ }^{1,2} \quad$ ZHANG Xin-Bo,"* \\ ('State Key Laboratory of Rare Earth Resource Utilization, Changchun Institute of Applied Chemistry, Changchun 130022, P. R. China; \\ ${ }^{2}$ University of Chinese Academy of Sciences, Beijing 100049, P. R. China)
}

\begin{abstract}
Supercapacitors (SCs) have been explored as one of the electrical sources because of their fast charge and discharge rates, good safety, and long cycle life. However, the limited energy densities of SCs hinder their further application. Thus, current research on SCs focuses on increasing their energy density. Enhancing specific capacitance is an effective way to increase energy density. In this review, we describe several approaches to achieve superior electrochemical properties by optimizing electrode materials and electrolytes. Considering electrode materials, their electrochemical performance is related to their specific surface area, pore structure, and electroconductivity. On one hand, the optimization of specific surface area and pore structure can increase their content of exposed active sites as well as electrolyte ion conductivity, which is beneficial for improved specific capacitance. On the other hand, enhanced electroconductivity leads to higher specific capacitance. The specific capacitances of electric double-layer capacitors and pseudocapacitors have been increased by optimizing carbon-based materials and metal hydroxides/oxides, respectively. Moreover, specific capacitance can be further enhanced by adding a redox mediator to the electrolyte as a pseudocapacitive source. This review offers perspectives to aid the development of next-generation supercapacitors with high specific capacitance.
\end{abstract}

Key Words: Supercapacitor; Specific capacitance; Electrode material; Electrolyte; ELectrical doublelayer capacitor; Pseudocapacitor

Received: July 7, 2016; Revised: November 1, 2016; Published online: November 1, 2016.

${ }^{*}$ Corresponding author. Email: xbzhang@ciac.ac.cn; Tel: +86-431-85262340.

The project was supported by the National Natural Science Foundation of China $(21422108,21271168,51472232)$.

国家自然科学基金 $(21422108,21271168,51472232)$ 资助项目

(c) Editorial office of Acta Physico-Chimica Sinica 


\section{1 引言}

超级电容器是一种常见的电化学储能体系, 具有充放电效率高、工作温度范围宽、使用寿命 长、绿色环保等独特的优点 ${ }^{1,2}$ 。近年来, 超级电容 器已被广泛应用于人们日常生活的方方面面如刹 车体系、后备电源等 ${ }^{3}$ 。然而, 由于超级电容器相 对有限的能量密度使其应用范围受到限制。因 此, 大量的研究人员深切关注保持其现有特点的 同时能提高其能量密度。根据能量密度 $E=C V^{2} / 2$ (其中, $C$ 是比容量, $V$ 是电位窗), 可以看出提高 比容量是一种行之有效的方法 ${ }^{4}$ 。

通常而言, 超级电容器可以根据其储能机理 分为两种类型: 双电层电容器和噟电容器 5 。双电 层电容器是利用电极/电解液界面上电荷分离所产 生的双电层来储存能量 6,7 。而噟电容器是利用电极 表面(或亚表面)的快速可逆氧化还原反应来储存能 量 89 。电极材料的特性对电容器的比容量起着至关 重要的作用 ${ }^{10,11}$ 。超级电容器的电极材料主要有碳 材料和氧化物/氢氧化物。碳材料由于其高比表面 积和良好的导电性, 常作为双电层电容的电极材 料如石墨烯和碳纳米管 ${ }^{12,13}$ 。金属氧化物/氢氧化物 (如氢氧化镍、氧化镍) 遵循噟电容机理 ${ }^{14}$, 常被用 作赝电容器电极材料 ${ }^{15,16}$ 。

\section{2 高容量超级电容器的制备}

通过控制电极材料的孔道结构和表面特征可 以改变比容量的大小 ${ }^{17,18}$ 。大量的研究表明通过改 善电极材料的比表面积、孔道结构和导电性可以 达到提高其电化学性能的目的 ${ }^{19,20}$, 。这是由于高比 表面积和孔道结构提供了更多的活性位点, 有利 于电解液离子的有效传输 ${ }^{21,22}$ 。而良好的导电性有 利于电子的快速运输以适应快速充放电过程 ${ }^{23}$ 。

\section{1 碳材料的优化}

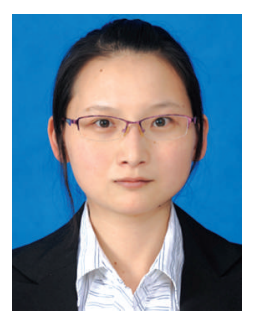

吴中, 1987 年生。2010年本科毕 业于安徽师范大学化学教育专业。 2010 年至 2016 年在中国科学院长 春应用化学研究所无机化学专业攻 读博士学位, 师从张新波研究员。 主要研究方向是无机纳米材料的合 成及其电化学性能测试, 尤其是金属氧化物材料 和碳材料的制备与其在超级电容器中的应用。
石墨烯是碳材料的一种, 具有独特的二维结 构和较高的比表面积, 近年来在能源领域得到广 泛关注 ${ }^{24,25}$ 。石墨烯可以通过多种合成途径得到, 尤其是氧化还原法被广泛用于石墨烯的制备。氧 化还原法是利用酸氧化和剥离石墨再在加热或者 还原剂的条件下被还原得到石墨烯 ${ }^{26,27}$ 。经过氧化 还原方法得到的石墨烯含有一些亲水官能团, 使 得电极材料更好地浸润在电解液中, 提高了电极/ 电解液的接触面积 ${ }^{28,29}$ 。然而, 石墨烯结构不稳 定, 层间的范德华力极易造成团聚形成多层石墨 烯, 从而减少了其比容量。

为了提高石墨烯材料的比容量, 以石墨烯和 酚醛树脂作为碳凝胶的前聚体在泡沫镍表面原位 合成了具有多孔结构的碳凝胶/泡沫镍, 如图 1 所 示 ${ }^{30}$ 。泡沫镍的表面在沉积碳凝胶前后发生了明显 的变化, 在扫描电镜下能看到表面碳凝胶是有碳 凝胶之间互相连接形成了三维大孔网络结构, 形 成了有效的电子传输和离子传输通道(图 1(a))。制 备得到的多孔碳凝胶/泡沫镍一体化电极可以直接 用作电极, 测试其在碱性电解液 $\left(6 \mathrm{~mol} \cdot \mathrm{L}^{-1} \mathrm{KOH}\right)$ 和有机电解液 $\left(1 \mathrm{~mol} \cdot \mathrm{L}^{-1} \mathrm{TEABF}_{4} / \mathrm{AN}\right.$ (四氟硼酸四 乙胺/乙腈))中的电化学性能。图 1(b)为碳凝胶/泡 沫镍电极在碱性电解液中的循环伏安曲线。在扫 速较小时, 循环伏安曲线均为较为规则的矩形。 当扫描速率增大, 循环伏安曲线出现较为严重的 偏移。这可能是由于扫描速度过快而电极/电解液 界面没有呈现及时吸脱附过程出现的极化状态。

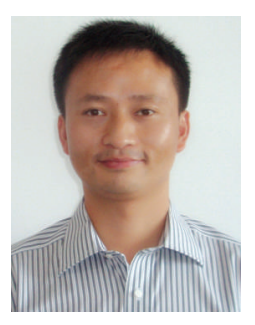

张新波, 1978 年生。1999 年在长 春科技大学获学士学位, 2005 年在 中国科学院长春应用化学研究所获 博士学位。2005-2009年在日本国 立产业技术综合研究所从事科学研 究(JSPS 和 NEDO 博士后)。2009年 12 月受聘中国科学院长春应用化学研究所稀土资 源利用国家重点实验室研究员、博士生导师。2011 年获中国科学院 “百人计划” 择优支持, 2014 年 获国家自然科学基金委 “优秀青年基金” 支持, 2015 年入选国家 “万人计划” 青年拔尖人才支持 计划。申请国家发明专利 16 项(6 项已授权), 日本 专利 2 项。承担中国科学院战略性先导科技专项、 国家自然科学基金、国家重点研发计划等课题。 

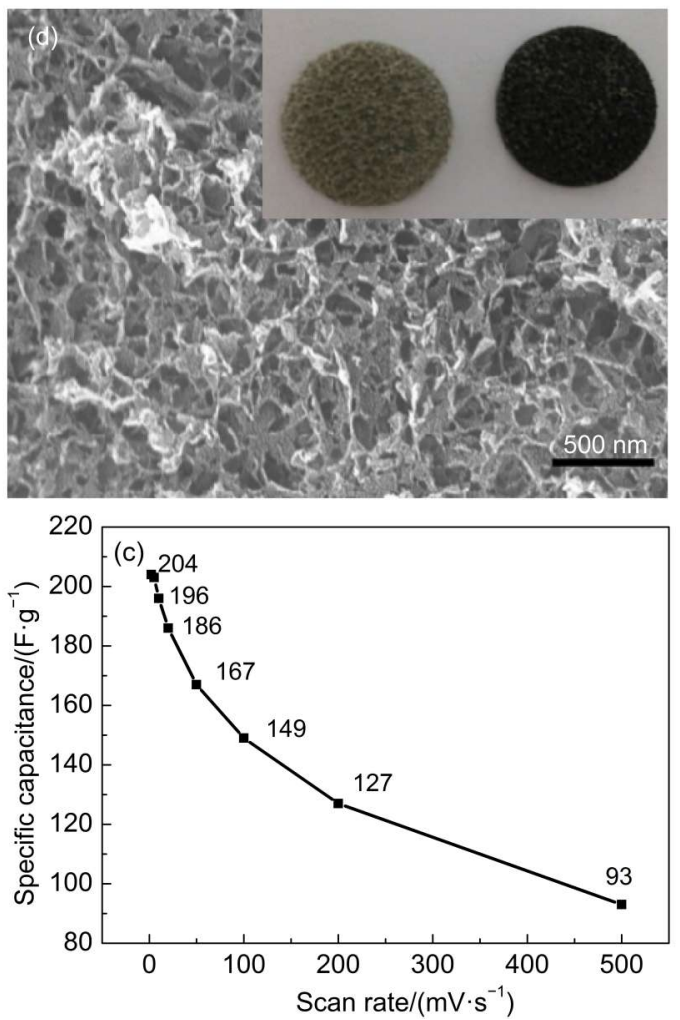
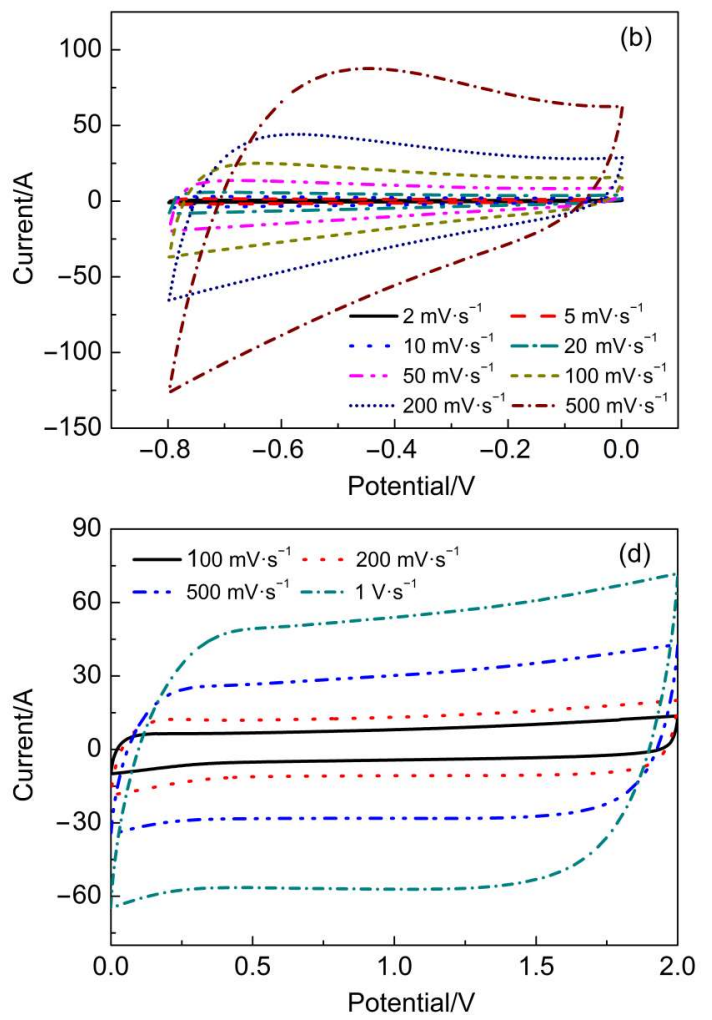

图 1 (a)泡沫镍表面的碳凝胶的扫描电镜(SEM)图像(插入图是沉积碳凝胶前后泡沫镍的外观照片);

(b) 碳凝胶/泡沫镍电极在 $6 \mathrm{~mol} \cdot \mathrm{L}^{-1} \mathrm{KOH}$ 中的循环伏安曲线; (c)碳凝胶/泡沫镍电极在 $6 \mathrm{~mol} \cdot \mathrm{L}^{-1} \mathrm{KOH}$ 中的比容量曲线; (d) 碳凝胶/泡沫镍电极在 $1 \mathrm{~mol} \cdot \mathrm{L}^{-1} \mathrm{TEABF}_{4} / \mathrm{AN}$ 中的循环伏安曲线 ${ }^{30}$

Fig.1 (a) SEM image of the as-prepared carbon aerogel on Ni foam (inset is the photo image of Ni foam before and after the deposition of carbon aerogel); (b) cyclic voltammetry $(\mathrm{CV})$ curves of carbon aerogel/Ni foam in $6 \mathrm{~mol} \cdot \mathrm{L}^{-1} \mathrm{KOH}$;

(c) variation in the specific capacitance of carbon aerogel/Ni foam as a function scan rate in $6 \mathrm{~mol} \cdot \mathrm{L}^{-1} \mathrm{KOH}$;

(d) $\mathrm{CV}$ curves of carbon aerogel/Ni foam in $1 \mathrm{~mol} \cdot \mathrm{L}^{-1} \mathrm{TEABF}_{4} / \mathrm{AN}^{30}$

$\mathrm{TEABF}_{4} / \mathrm{AN}$ : tetraethylammonium tetrafluoroborate/acetonitrile

图 1(c)总结了碳凝胶/泡沫镍电极在不同扫描速率 下的比容量变化图。根据图中曲线, 可以发现在 碱性电解液中碳凝胶/泡沫镍一体化电极最高可以 得到达 $204 \mathrm{~F} \cdot \mathrm{g}^{-1}$ 的比容量。不仅如此, 随着扫描 速率的不断增大, 比电容的减小缓慢, 在扫描速 率高达 $200 \mathrm{mV} \cdot \mathrm{s}^{-1}$ 时, 仍可以得到 $127 \mathrm{~F} \cdot \mathrm{g}^{-1}$ 的比 容量。图 $1(\mathrm{~d})$ 是碳凝胶/泡沫镍电极在有机电解 液—- $\mathrm{TEABF}_{4} / \mathrm{AN}$ 中的循环伏安曲线, 说明了其 在有机电解液中理想的双电层电容行为。

多孔碳凝胶/泡沫镍电极在碱性电解液和有机 电解液中表现出了高的比容量得益于其本身的电 极特点: (1) 得到的三维碳结构不仅可以抑制石墨 烯层间的团聚从而获得更多的活性位点而且其构 建的孔道结构有利于提高离子传导率; (2) 泡沫镍 的加入避免了不导电的粘接剂的加入, 有利于提 高电极材料的电子传导率。因此得到的多孔碳凝 胶/泡沫镍一体化电极具有良好的比容量。
提高碳材料的比容量, 还可以通过在双电层 电容器体系中引入氧化还原电解液来实现。这种 方法能得到更高的比容量是由于电解液的氧化还 原反应。这种氧化还原反应发生在电解液中而不 是电极材料上, 因此可以保证电极材料的稳定 ${ }^{31}$ 。 这种方法比较简单, 并且可以在提高比容量的同 时保持电极材料的稳定性。氧化还原电解液被应 用于双电层电解液中来提高比容量的方法已被大 量文献报道 ${ }^{32}$ 。选取 $0.09 \mathrm{~mol} \cdot \mathrm{L}^{-1} \mathrm{CuCl}_{2}+1 \mathrm{~mol}$. $\mathrm{L}^{-1} \mathrm{HNO}_{3}$ 为氧化还原电解液加入双电层体系中, 电 极材料是以氧化石墨、苯胺、间苯二酚和甲醛为原 料合成的氮掺杂的多孔碳二维材料 (NOPCSs) ${ }^{33}$ 。电 极材料有如下的优点: (1) 二维结构有利于形成稳 定的双电层并具有充足的电极/电解液接触面 ${ }^{34}$; （2）多孔结构有利于离子的快速运输; (3) 杂原子的 存在不仅使得其在电解液中的浸润性更好, 而且 杂原子中的孤对电子增加了电极材料的导电性, 
从而提高了电极材料的比容量 ${ }^{35,36}$ 。对比材料为相 同实验条件下不加入石墨烯合成的碳材料 CNS。

首先, 通过扫描电镜和透射电镜来直观的观 察碳材料 NOPCSs 样品表面的微观形貌。从图 2(a, b) 可以看出得到的碳材料 NOPCSs 是片状二维结 构, 并含有多孔结构。不仅如此, 通过 X射线光 电子能谱(XPS)的方法可以分析 NOPCSs 材料中氮 掺杂的存在。图 2(c) 是 $\mathrm{N} 1 s$ 的拟合曲线, 表明 NOPCSs 中的氮掺杂有三种方式: 吡啶氮( ( $397.2 \pm$ $0.1) \mathrm{eV})$, 吡咯氮 $((400.0 \pm 0.1) \mathrm{eV})$ 和氧化氮 $((405.1 \pm 0.1) \mathrm{eV})$ 。在三电极体系中测试所得材料 的电化学性能。电解液分别为酸性电解液 $(1 \mathrm{~mol}$ $\left.\mathrm{L}^{-1} \mathrm{H}_{2} \mathrm{SO}_{4}\right)$ 和氧化还原电解液 $\left(0.09 \mathrm{~mol} \cdot \mathrm{L}^{-1} \mathrm{CuCl}_{2}+\right.$ $\left.1 \mathrm{~mol} \cdot \mathrm{L}^{-1} \mathrm{HNO}_{3}\right)$ 。首先, 将制备得到的电极材料 NOPCSs 和 CNS 在 $1 \mathrm{~mol} \cdot \mathrm{L}^{-1} \mathrm{H}_{2} \mathrm{SO}_{4}$ 中测试其储能机 理。图 2(d) 是 NOPCSs 和 CNS 在扫描速率为 20 $\mathrm{mV} \cdot \mathrm{s}^{-1}$ 时的循环伏安曲线。根据循环伏安曲线的 形状可以看到它们都是标准的矩形因此可以判断 它们都遵从双电层电容储能机理。根据循环伏安 曲线可以计算得到 NOPCSs 在不同扫描速率下的比 容量。在扫描速率为 $20 \mathrm{mV} \cdot \mathrm{s}^{-1}$ 时, NOPCSs 的比 容量为 $107.2 \mathrm{~F} \cdot \mathrm{g}^{-1}$ 。
而测试NOPCSs 在氧化还原电解液中的电化学 性能与其在酸性电解液中完全不同。图 2(e) 是 NOPCSs 在不同扫描速率下的循环伏安曲线。根据 循环伏安曲线的形状可以看到它们有氧化还原峰 的出现, 因此可以判断有氧化还原反应的参与。 根据循环伏安曲线可以计算得到在不同扫描速率 下的比容量, 如图 2(f) 所示。NOPCSs 在扫描速率 为 $2 \mathrm{mV} \cdot \mathrm{s}^{-1}$ 表现出最高的比容量为 $5073.5 \mathrm{~F} \cdot \mathrm{g}^{-1}$ 。 图 2(f) 的插入图是 NOPCSs 和 CNS 在扫描速率为 $20 \mathrm{mV} \cdot \mathrm{s}^{-1}$ 时的循环伏安曲线。此时的 NOPCSs 的 比容量为 $1304.3 \mathrm{~F} \cdot \mathrm{g}^{-1}$, 是其在酸性电解质中容量 的 100 倍。由此可以看到氧化还原电解液的加入极 大的提高了双电层电容器的比容量, 这是一种更 为简便的提高双电层电容器比容量的方法。

\section{2 氧化物/氢氧化物的优化}

氢氧化镍 $\left(\mathrm{Ni}(\mathrm{OH})_{2}\right)$ 因具有独特的二维层状结 构, 较高的理论比容量 $\left(2675 \mathrm{~F} \cdot \mathrm{g}^{-1}\right)$, 在噟电容电 极材料的研究中备受关注。 $\mathrm{Ni}(\mathrm{OH})_{2}$ 的赝电容来 自 $\mathrm{Ni}^{2+} / \mathrm{Ni}^{3+}$ 的转化 ${ }^{37}: \mathrm{Ni}(\mathrm{OH})_{2}+\mathrm{OH}^{-}=\mathrm{NiOOH}+$ $\mathrm{H}_{2} \mathrm{O}+\mathrm{e}^{-}$。然而, 由于氢氧化镍的导电性较差导致 传输电子的能力有限, 实验中得到的氢氧化镍的 比容量远低于其理论值。为了提高 $\mathrm{Ni}(\mathrm{OH})_{2}$ 的电化
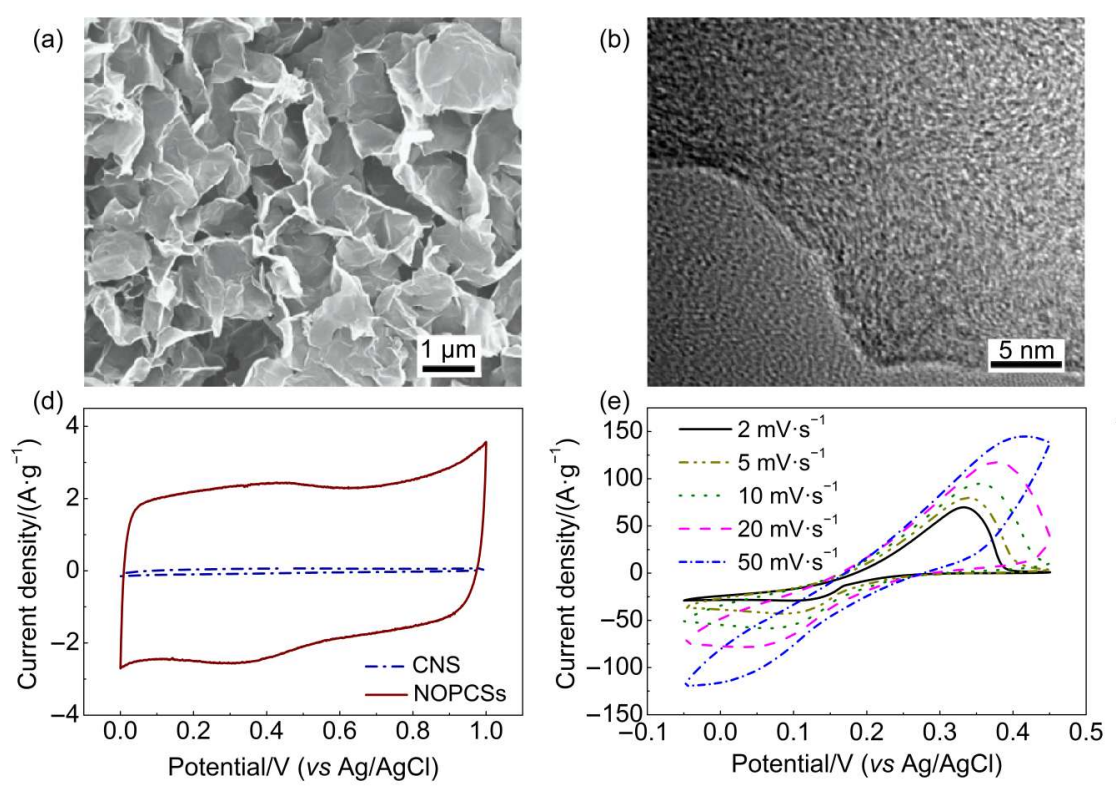
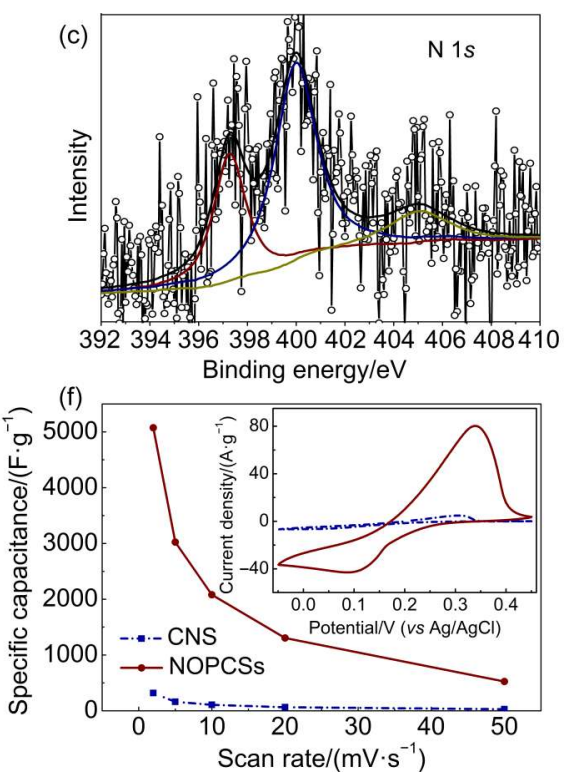

图 2 NOPCSs 的(a) SEM 和(b) TEM 图像; (c) NOPCSs 的 N $1 s$ 的拟合曲线; (d) NOPCSs 和 CNS 在 $1 \mathrm{~mol} \cdot \mathrm{L}^{-1} \mathrm{H}_{2} \mathrm{SO}_{4}$ 电解液 中的循环伏安曲线(扫描速率为 $20 \mathrm{mV} \cdot \mathrm{s}^{-1}$ ); 在氧化还原电解液中, (e) NOPCSs 在不同扫描速率下的循环伏安曲线和 (f) NOPCSs 和 CNS 在不同扫描速率下的比容量(插入图为 NOPCSs 和 CNS 在扫描速率为 $20 \mathrm{mV} \cdot \mathrm{s}^{-1}$ 时的循环伏安曲线)

Fig.2 (a) SEM and (b) TEM images of NOPCSs; (c) deconvolution of $\mathrm{N} 1 \mathrm{~s}$ spectrum of NOPCSs; (d) CV curves of the NOPCSs and CNS at a scan rate of $20 \mathrm{mV} \cdot \mathrm{s}^{-1}$ in $1 \mathrm{~mol} \cdot \mathrm{L}^{-1} \mathrm{H}_{2} \mathrm{SO}_{4}$; in the redox-active electrolyte, (e) $\mathrm{CV}$ curves of NOPCSs and (f) variation of specific capacitances with scan rates of NOPCSs and CNS (inset plots are CV curves of NOPCSs and CNS at a scan rate of $\left.20 \mathrm{mV} \cdot \mathrm{s}^{-1}\right)^{33}$ 
学性能, 构造金属氢氧化物和碳材料的复合物是 一种有效的方法。利用氢氧化镍和石墨烯间的静 电吸引作用将 $\mathrm{Ni}(\mathrm{OH})_{2}$ 和石墨烯复合。由于其相互 作用, $\mathrm{Ni}(\mathrm{OH})_{2}$ 被吸附在石墨烯的表面并沿着其表 面生长从而实现了均匀复合并得到了片状的氢氧 化镍复合材料, 如图 3(a)所示 ${ }^{38}$ 。为了研究石墨烯 加入的多少对其比容量的影响, 实验中测试了三 种不同比例的复合物的比容量。石墨烯的量由少 到多的三种复合物依次标记为 $\mathrm{Ni}(\mathrm{OH})_{2} / \mathrm{GS}-20$ 、 $\mathrm{Ni}(\mathrm{OH})_{2} / \mathrm{GS}-5$ 和 $\mathrm{Ni}(\mathrm{OH})_{2} / \mathrm{GS}-2$ 。为了研究石墨烯的 加入对 $\mathrm{Ni}(\mathrm{OH})_{2}$ 电化学性能的影响, 在保证其他实 验条件相同的情况下不加入石墨烯合成得到了对 比材料纯氢氧化镍。

通过 X射线衍射(XRD)谱图表征了合成得到的 前聚体、不同比例的 $\mathrm{Ni}(\mathrm{OH})_{2} /$ 石墨烯复合材料和对 比材料的晶型结构, 如图 3(b,c) 所示。可以通过衍 射峰的强度和位置定性的判断在复合前后氢氧化 镍的晶型发生了改变。不仅如此, 不同比例的复 合物的图谱可以看出随着石墨烯的加入量的增多 衍射峰逐渐变强, 结晶性越好。通过透射电镜
(TEM) 表征三种不同比例的 $\mathrm{Ni}(\mathrm{OH})_{2} / \mathrm{GS}$ 复合材料的 形貌特征, 如图 3(d-f)所示。从图 3(d)中可以看到 当石墨烯较少时, $\mathrm{Ni}(\mathrm{OH})_{2} / \mathrm{GS}-20$ 的表面有部分团 聚在一起的氢氧化镍颗粒。增加石墨烯的量得到 复合材料 $\mathrm{Ni}(\mathrm{OH})_{2} / \mathrm{GS}-5$ (图 3(e)), 发现其为片状结 构, 氢氧化镍在石墨烯的表面均匀覆盖。当继续 增加石墨烯的量得到 $\mathrm{Ni}(\mathrm{OH})_{2} / \mathrm{GS}-2$ 的形貌如图 3(f) 所示, 有过量的来源于石墨烯的褶皱结构。

通过循环伏安法和恒电流充放电法来确定电 极材料的储能机理以及表征电极材料的比容 量。图 4(a) 是复合材料 $\mathrm{Ni}(\mathrm{OH})_{2} / \mathrm{GS}-5$ 和对比材料 $\mathrm{Ni}(\mathrm{OH})_{2}$ 的循环伏安曲线图。从图中可以看到这两 种材料的循环伏安图都有一对氧化还原峰, 可以 判定这些电极材料的储能机理遵循赝电容机理。 通过其循环伏安曲线可以计算得到其在不同扫描 速率下的比容量, 如图 4(b)所示。复合材料相对于 对比材料表现出更高的比容量, 在扫描速率为 2 $\mathrm{mV} \cdot \mathrm{s}^{-1}$ 时 $\mathrm{Ni}(\mathrm{OH})_{2} / \mathrm{GS}-5$ 和 $\mathrm{Ni}(\mathrm{OH})_{2}$ 的比容量分别为 1503 和 $1064 \mathrm{~F} \cdot \mathrm{g}^{-1}$ 。复合材料相比于对比材料都表 现出了较高的比容量, 可以证明石墨烯的存在对
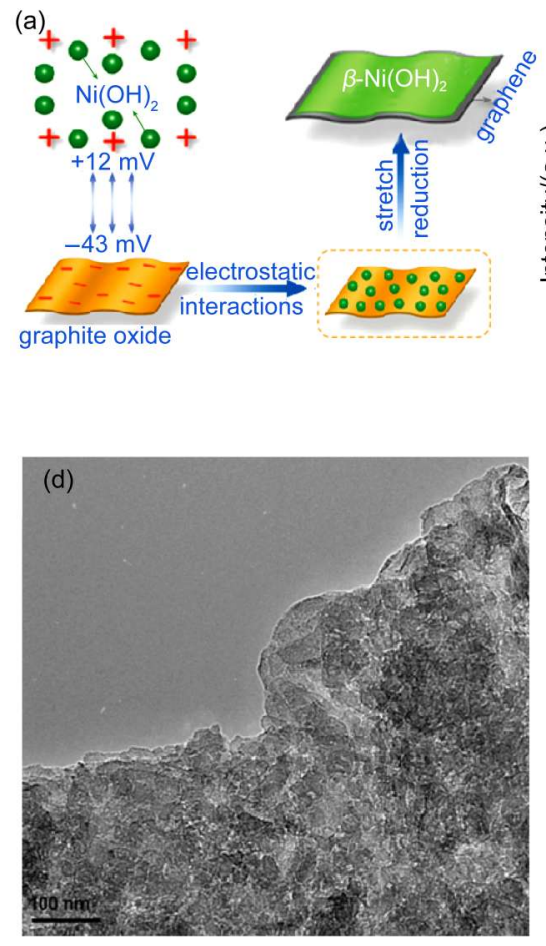
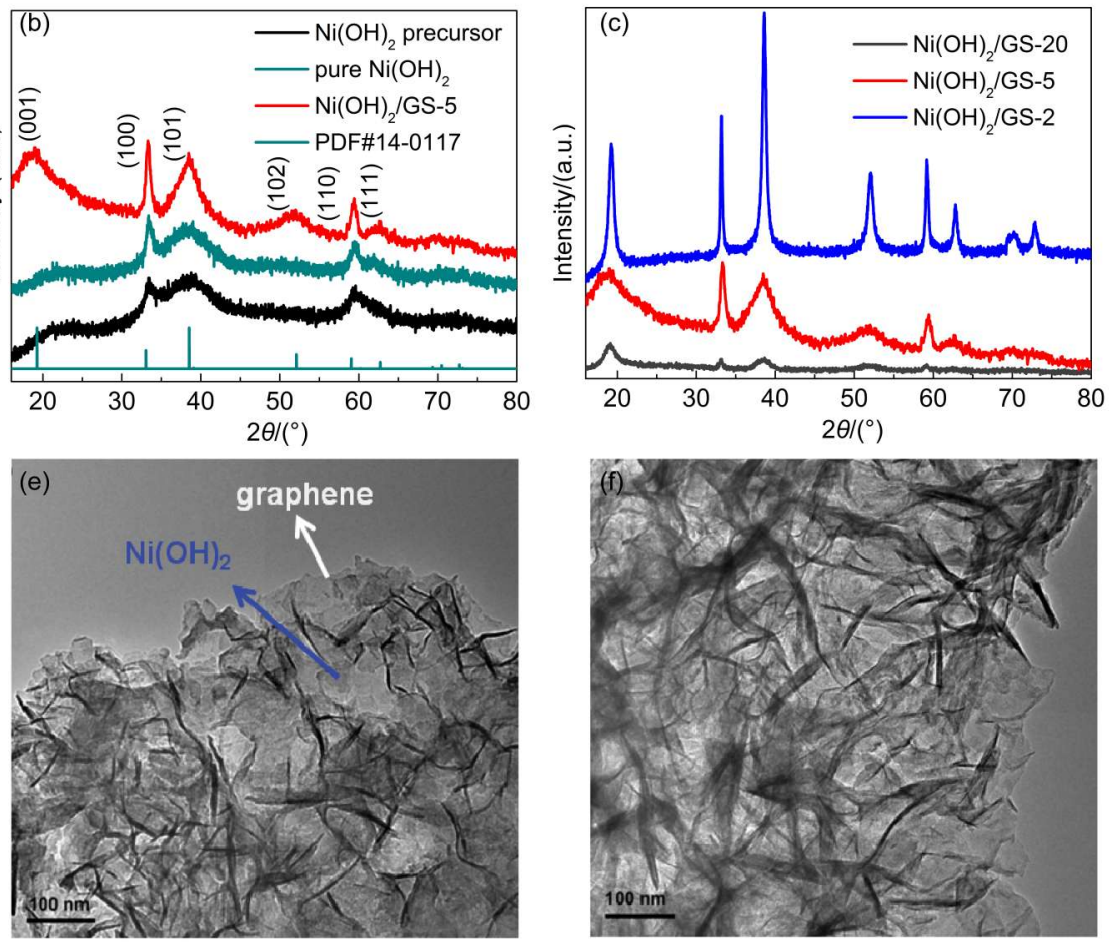

图 3 (a) $\mathrm{Ni}(\mathrm{OH})_{2} /$ 石墨烯复合材料制备过程示意图; (b)前聚体、对比材料和复合材料 $\mathrm{Ni}(\mathrm{OH})_{2} / \mathrm{GS}-5$ 的 XRD 谱图; (c)三种 不同比例的氢氧化镍与石墨烯复合物的 XRD 谱图; 三种不同比例的复合材料(d) $\mathrm{Ni}(\mathrm{OH})_{2} / \mathrm{GS}-20 、(\mathrm{e}) \mathrm{Ni}(\mathrm{OH})_{2} / \mathrm{GS}-5$ 、

(f) $\mathrm{Ni}(\mathrm{OH})_{2} / \mathrm{GS}-2$ 的 TEM 图像 ${ }^{38}$

Fig.3 (a) Schematic illustration of $\mathrm{Ni}(\mathrm{OH})_{2}$ nanoplates stretch growth on graphene; (b) XRD patterns of precursor $\mathrm{Ni}(\mathrm{OH})_{2}$, pure $\mathrm{Ni}(\mathrm{OH})_{2}$ and the $\mathrm{Ni}(\mathrm{OH})_{2} / \mathrm{GS}-5$ composite; (c) XRD patterns of the $\mathrm{Ni}(\mathrm{OH})_{2} / \mathrm{GS}$ composites; TEM images of (d) $\mathrm{Ni}(\mathrm{OH})_{2} / \mathrm{GS}-20$, (e) $\mathrm{Ni}(\mathrm{OH})_{2} / \mathrm{GS}-5$, (f) $\mathrm{Ni}(\mathrm{OH})_{2} / \mathrm{GS}-2$ composites $^{38}$ 

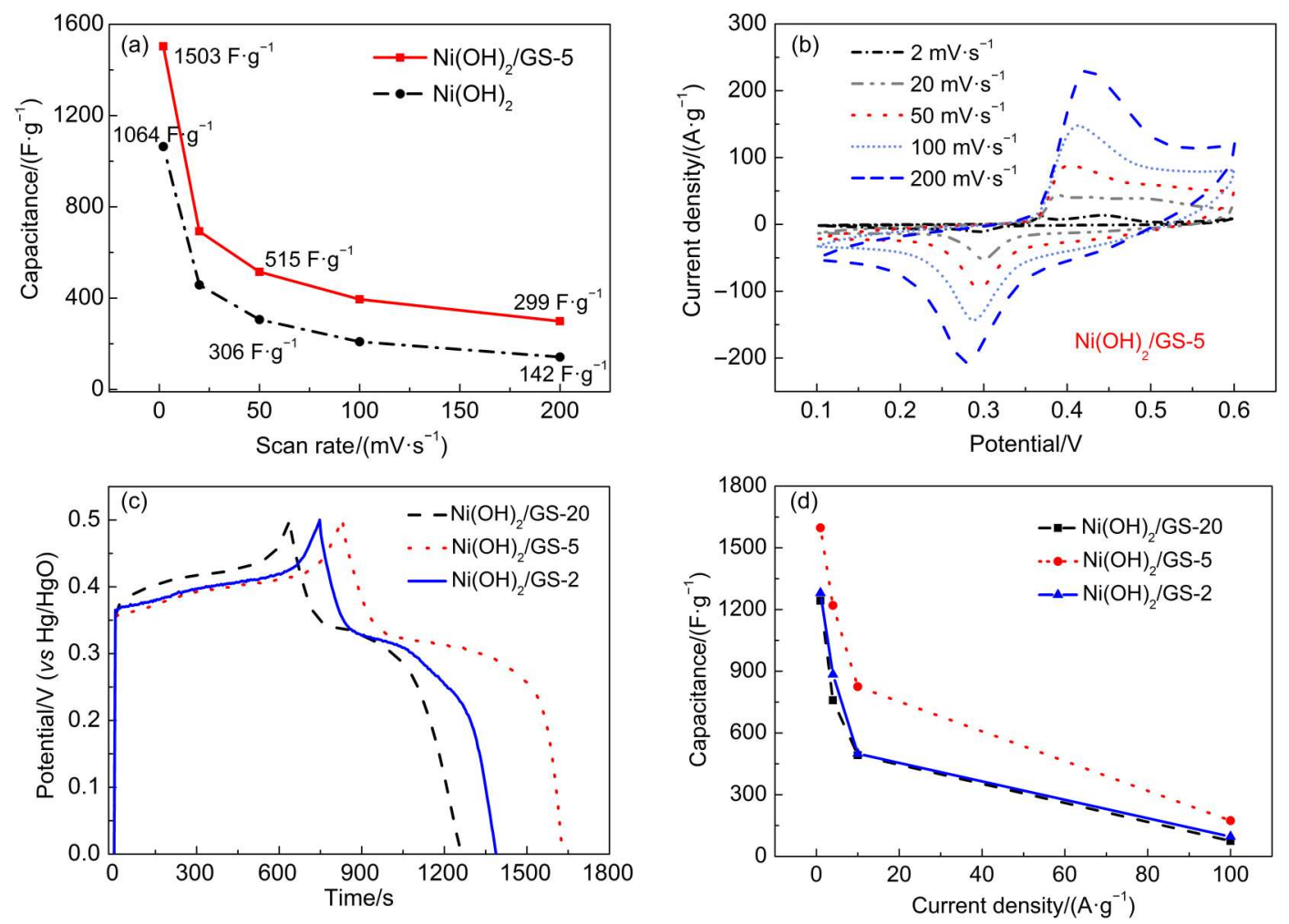

图 4 (a)复合材料 $\mathrm{Ni}(\mathrm{OH})_{2} / \mathrm{GS}-5$ 在不同的扫描速率下的循环伏安曲线图; (b)复合材料 $\mathrm{Ni}(\mathrm{OH})_{2} / \mathrm{GS}-5$ 和对比材料 $\mathrm{Ni}(\mathrm{OH})_{2}$ 的 容量相对于扫描速率的变化图; (c)三种不同比例的复合材料 $\mathrm{Ni}(\mathrm{OH})_{2} / \mathrm{GS}-20, \mathrm{Ni}(\mathrm{OH})_{2} / \mathrm{GS}-5, \mathrm{Ni}(\mathrm{OH})_{2} / \mathrm{GS}-2$ 在 $1 \mathrm{~A} \cdot \mathrm{g}^{-1}$ 的 电流密度下的恒电流充放电曲线图; (d)三种不同比例的复合材料的容量相对于电流密度的变化图 ${ }^{38}$

Fig.4 (a) $\mathrm{CV}$ curves of $\mathrm{Ni}(\mathrm{OH})_{2} / \mathrm{GS}-5$; (b) average specific capacitances of $\mathrm{Ni}(\mathrm{OH})_{2} / \mathrm{GS}-5$ and pure $\mathrm{Ni}(\mathrm{OH})_{2}$ at various scan rates; (c) galvanostatic charge and discharge curves of $\mathrm{Ni}(\mathrm{OH})_{2} / \mathrm{GS}-20, \mathrm{Ni}(\mathrm{OH})_{2} / \mathrm{GS}-5$, and $\mathrm{Ni}(\mathrm{OH})_{2} / \mathrm{GS}-2$ at $1 \mathrm{~A} \cdot \mathrm{g}^{-1}$;

(d) average specific capacitances of $\mathrm{Ni}(\mathrm{OH})_{2} / \mathrm{GS}-20, \mathrm{Ni}(\mathrm{OH})_{2} / \mathrm{GS}-5$, and $\mathrm{Ni}(\mathrm{OH})_{2} / \mathrm{GS}-2$ at various current densities ${ }^{38}$

复合材料的积极作用。其比容量的提升得益于一 方面提高了电极材料的导电性从而提高了电子传 导能力; 另一方面更好地分散了氢氧化镍增加了 活性位点从而提高了离子传输能力。

通过恒电流充放电法测试了三种不同比例的 复合材料的比容量来比较加入石墨烯的多少对复 合材料电化学性能的影响。图 4(c) 是三种不同比例 的复合材料在电流密度为 $1 \mathrm{~A} \cdot \mathrm{g}^{-1}$ 时的充放电曲线 比较图。由图中可以看到它们的曲线形状相似, $\mathrm{Ni}(\mathrm{OH})_{2} / \mathrm{GS}-5$ 表现出较好的电化学性能。根据恒电 流充放电曲线可以计算得到电极材料的比容量, 如图 4(d)所示。不同的石墨烯的量会影响复合材料 的电化学性能。过少或过多的石墨烯都会导致团 聚现象, 对电极材料的电化学性能带来不利的影 响。可以通过优化石墨烯与氢氧化镍的比例来得 到更好的电化学性能。

氧化镍因具有较好的化学稳定性、良好的电 化学行为, 被用作超级电容器的电极材料。然而
由于氧化物本身的半导体性质, 其有限的导电性 限制了更多的应用 ${ }^{39-41}$ 。因此, 如何更大限度的发 挥氧化镍电极材料的噟电容性能仍然是一个非常 大的挑战。与上一章改进氢氧化镍的电化学性能 方法类似, 提高氧化镍电化学性能的方法主要 有: (1) 将氧化镍材料纳米化从而获得更多的活性 位点和电极/电解液接触面积 ${ }^{42,43}$; (2) 将氧化物材料 与碳材料复合改善导电性 ${ }^{44,45}$ 。其中, 碳纳米管是 一维碳材料, 具有良好的导电性和独特的纳米管 结构 ${ }^{46}$ 。另外, 碳纳米管是一种很好的基底, 常被 用于提高赝电容电极材料的导电性。

首先将碳纳米管经过氧化处理使其表面附上 一些含氧集团, 再通过静电作用将碳纳米管与氧 化镍前聚体复合。将复合物经过抽滤并冷冻干燥 可得到氧化镍前聚体/OCNT 膜材料。再经过煅烧 即可得到氧化镍/碳纳米管膜材料, 整个制备过程 如图 5(a) 所示 ${ }^{47}$ 。这种膜材料可以直接用于电极测 试, 不仅改进了电极制备过程而且避免了粘接剂 
(a)

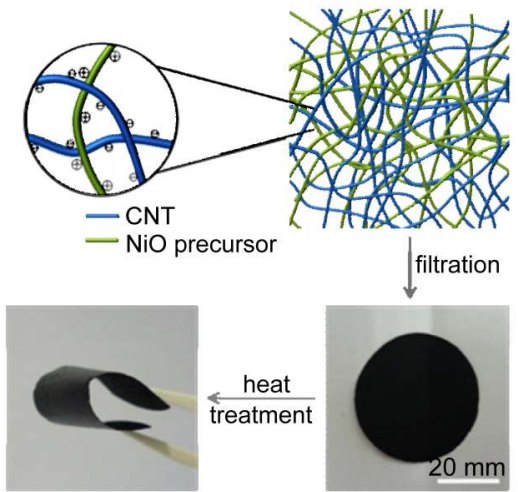

(c)

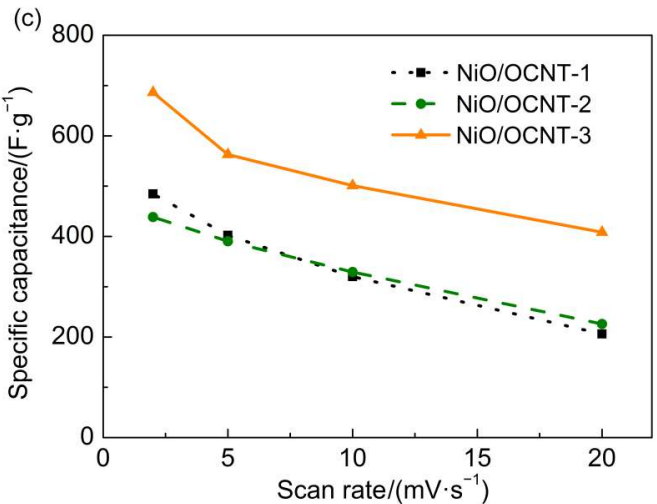

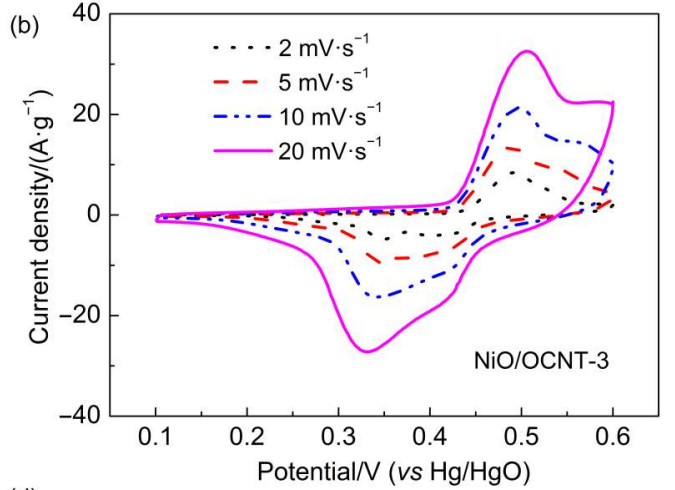

(d)

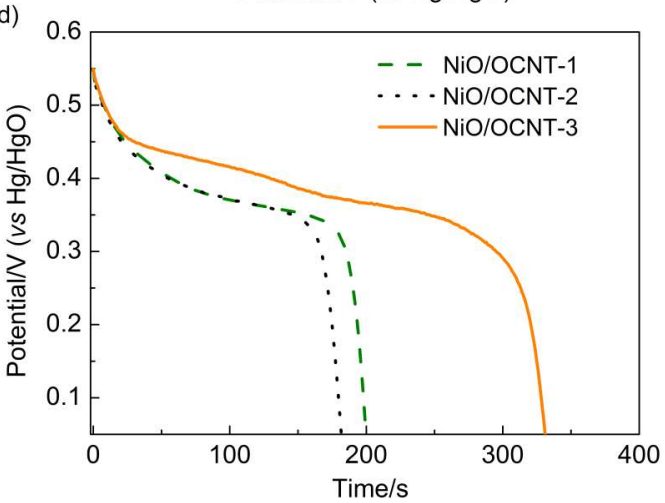

图 5 (a)氧化镍/碳纳米管膜材料的制备过程示意图; (b) NiO/OCNT-3 在不同扫描速率下的循环伏安曲线图;

(c)三种不同比例的复合膜材料根据循环伏安曲线计算得到的比容量比较图;

(d)三种不同比例的复合材料在 $1 \mathrm{~A} \cdot \mathrm{g}^{-1}$ 的电流密度下的恒电流放电曲线比较图 ${ }^{47}$

Fig.5 (a) Schematic representation of the synthesis of NiO/OCNT films; (b) CV curves of NiO/OCNT-3;

(c) average specific capacitances of the $\mathrm{NiO} / \mathrm{OCNT}$ composites at various scan rates;

(d) galvanostatic discharge curves of the $\mathrm{NiO} / \mathrm{OCNT}$ composites at $1 \mathrm{~A} \cdot \mathrm{g}^{-1} 47$

的加入，提高了整个电极的导电性从而提高了其 电容性能。为了对比加入的碳纳米管的量对复合 膜材料的影响，同时制备了含有不同比例的氧化 镍和碳纳米管的复合材料。本文制备的复合材料 中氧化镍与碳纳米管的质量比分别为 $2: 1 、 1: 1$ 和 $1: 2$, 相应的复合膜材料分别被命名为 $\mathrm{NiO} / \mathrm{OCNT}-$ 1、 $\mathrm{NiO} / \mathrm{OCNT}-2$ 和 $\mathrm{NiO} / \mathrm{OCNT}-3$ 。

制备得到的不同比例的氧化镍/碳纳米管膜材 料可以直接用于电极测试。通过循环伏安曲线来 确定复合材料的电荷储存机理, 如图 5(b) 所示, 有 一对明显的氧化还原峰出现。这一结果表明氧化 镍/碳纳米管膜的储能机理遵从噟电容机理。图 5 (c)展示了三种不同比例的复合膜材料根据循环伏 安曲线计算得到的比容量。可以发现 $\mathrm{NiO} / \mathrm{OCNT}-3$ 比 $\mathrm{NiO} / \mathrm{OCNT}-1$ 和 $\mathrm{NiO} / \mathrm{OCNT}-2$ 具有更高的比容 量。以扫描速率为 $2 \mathrm{mV} \cdot \mathrm{s}^{-1}$ 为例, $\mathrm{NiO} / \mathrm{OCNT}-3$ 的 比容量为 $686.5 \mathrm{~F} \cdot \mathrm{g}^{-1}$, 而 $\mathrm{NiO} / \mathrm{OCNT}-1$ 和 $\mathrm{NiO} /$ OCNT- 2 的比容量分别为 484.5 和 $438.4 \mathrm{~F} \cdot \mathrm{g}^{-1}$ 。进一 步通过测试三种不同比例的复合材料在同一电流
密度下(图 5(d)) 的恒电流放电曲线证实了这一结论。 碳纳米管的加入改善了氧化镍材料的电化学 性能, 可能有以下几个方面原因: (1) 碳纳米管的 加入在复合材料中作为导电骨架增加了电极材料 的导电性; (2) 制备得到的膜材料可以直接被应用 为电极, 避免了粘接剂的加入对电极材料导电性 的损失; (3) 加入不同量的碳纳米管对复合材料的 电化学性能有至关重要的影响。通过调节氧化镍 和碳纳米管的比例可以优化其复合材料的电化学 性能。

此外, 双金属氧化物和双金属硫化物因其具 有比单金属氧化物更高的电子导电性和更高的比 电容, 已成为近几年研究的热点 ${ }^{48}$ 。例如, 镍钴氧 化物和镍钴硫化物已经在实验中证明它们具有良 好的电化学性能, 是两类有效的赝电容材料 ${ }^{49,50}$ 。

\section{3 结论与展望}

通过理解超级电容器的储能机理, 设计和研 究具有合理结构的电极材料来提高其电化学性 
能。对赝电容和双电层电容的研究发现：(1) 将赝 电容材料与碳材料复合可以改善其电化学性能。 (2) 增加碳材料和电解液的接触面积可以有效改善 其电化学性能。另外, 氧化还原电解液的加入可 以极大地提高双电层电容器的比容量。(3) 一体化 电极的制备可以避免常规电极制备过程中不导电 的粘接剂的加入, 可以有效提高电极材料的导电 性从而提高其电化学性能。本文主要通过提高电 极材料的比容量来达到提高能量密度的目的, 并 且取得了一定的成功。但仍有一些工作可以用来 进一步提高电极材料的能量密度。如将赝电容材 料和双电层材料进行匹配构建不对称电容器, 这 样可以极大的提升整个体系的电位窗, 从而达到 提高能量密度的目的。

\section{References}

(1) Kuang, Q.; Jiang, Z. Y.; Xie, Z. X.; Zheng, L. Acc. Chem. Res 2014, 47, 308. doi: 10.1021/ar400092x

(2) Jing, M. J.; Hou, H. S.; Yang, Y. C.; Zhu, Y. R.; Wu, Z. B.; Ji, X. B. Electrochim. Acta 2015, 165, 198. doi: 10.1016/j. electacta.2015.03.032

(3) Burke, A. J. Power Sources 2000, 91, 37. doi: 10.1016/S03787753(00)00485-7

(4) Yu, D.; Qian, Q.; Wei, L.; Jiang, W.; Goh, K.; Wei, J.; Zhang, J.; Chen, Y. Chem. Soc. Rev. 2015, 44, 647. doi: 10.1039/ C4CS00286E

(5) Simon, P.; Gogotsi, Y. Nat. Mater. 2008, 7, 845. doi: 10.1038/ nmat2297

(6) Ashok Kumar, N.; Baek, J. B. Chem. Commun. 2014, 50, 6298. doi: 10.1039/C4CC01049C

(7) Jiang, H.; Lee, P. S.; Li, C. Energy Environ. Sci. 2013, 6, 41. doi: 10.1039/C2EE23284G

(8) Rakhi, R. B.; Chen, W.; Cha, D.; Alshareef, H. N. Adv. Energy Mater. 2012, 2, 381. doi: 10.1002/aenm.201100609

(9) Xia, X.; Tu, J.; Zhang, Y.; Chen, J.; Wang, X.; Gu, C.; Guan, C.; Luo, J.; Fan, H. J. Chem. Mater. 2012, 24, 3793. doi: 10.1021/ cm302416d

(10) Augustyn, V.; Simon, P.; Dunn, B. Energy Environ. Sci. 2014, 7, 1597. doi: 10.1039/C3EE44164D

(11) Yu, M.; Huang, Y.; Li, C.; Zeng, Y.; Wang, W.; Li, Y.; Fang, P.; Lu, X.; Tong, Y. Adv. Funct. Mater. 2015, 25, 324. doi: 10.1002/ adfm.201402964

(12) Kaempgen, M.; Chan, C. K.; Ma, J.; Cui, Y.; Gruner, G. Nano Lett. 2009, 9, 1872. doi: 10.1021/n18038579

(13) Zhang, L. L.; Zhou, R.; Zhao, X. S. J. Mater. Chem. 2010, 20, 5983. doi: $10.1039 / \mathrm{C} 000417 \mathrm{~K}$

(14) Jing, M. J.; Wang, C. W.; Hou, H. S.; Wu, Z. B.; Zhu, Y. R.; Yang, Y. C.; Jia, X. N.; Zhang, Y.; Ji, X. B. J. Power Sources
2015, 298, 241. doi: 10.1016/j.jpowsour.2015.08.039

(15) Chen, H.; Hu, L.; Yan, Y.; Che, R.; Chen, M.; Wu, L. Adv. Energy Mater. 2013, 3, 1636. doi: 10.1002/aenm.201300580

(16) Su, D.; Kim, H. S.; Kim, W. S.; Wang, G. Chemistry 2012, 18 , 8224. doi: 10.1002/chem.201200086

(17) Liu, C.; Li, F.; Ma, L. P.; Cheng, H. M. Adv. Mater. 2010, 22, E28. doi: 10.1002/adma.200903328

(18) Hall, P. J.; Mirzaeian, M.; Fletcher, S. I.; Sillars, F. B.; Rennie, A. J. R.; Shitta-Bey, G. O.; Wilson, G.; Cruden, A.; Carter, R. Energy Environ. Sci. 2010, 3, 1238. doi: 10.1039/C0EE00004C

(19) Chaudhuri, R. G.; Paria, S. Chem. Rev. 2012, 112, 2373. doi: $10.1021 /$ cr100449n

(20) Lee, S. W.; Gallant, B. M.; Byon, H. R.; Hammond, P. T.; ShaoHorn, Y. Energy Environ. Sci. 2011, 4, 1972. doi: 10.1039/ C0EE00642D

(21) Fang, B.; Kim, J. H.; Kim, M. S.; Bonakdarpour, A.; Lam, A.; Wilkinson, D. P.; Yu, J. S. J. Mater. Chem. 2012, 22, 19031. doi: 10.1039/C2JM33435F

(22) Wu, Z. B.; Pu, X. L.; Ji, X. B.; Zhu, Y. R.; Jing, M. J.; Chen, Q. Y.; Jiao, F. P. Electrochim. Acta 2015, 174, 238. doi: 10.1016/j. electacta.2015.06.011

(23) Arruda, T. M.; Heon, M.; Presser, V.; Hillesheim, P. C.; Dai, S.; Gogotsi, Y.; Kalinin, S. V.; Balke, N. Energy Environ. Sci. 2013, 6, 225. doi: 10.1039/C2EE23707E

(24) Chen, S.; Liu, Y.; Chen, J. Chem. Soc. Rev. 2014, 43, 5372. doi: $10.1039 / \mathrm{C} 4 \mathrm{CS} 00087 \mathrm{~K}$

(25) Zhang, J.; Zhao, X. S. ChemSusChem 2012, 5, 818. doi: 10.1002/cssc.201100571

(26) Xu, Y.; Chen, C. Y.; Zhao, Z.; Lin, Z.; Lee, C.; Xu, X.; Wang, C.; Huang, Y.; Shakir, M. I.; Duan, X. Nano Lett. 2015, 15, 4605. doi: 10.1021/acs.nanolett.5b01212

(27) Zhu, J.; Yang, D.; Yin, Z.; Yan, Q.; Zhang, H. Small 2014, 10, 3480. doi: $10.1002 / \mathrm{smll} .201303202$

(28) Peng, L.; Xu, Z.; Liu, Z.; Wei, Y.; Sun, H.; Li, Z.; Zhao, X.; Gao, C. Nat. Commun. 2015, 6, 5716. doi: 10.1038/ncomms6716

(29) Wang, L. J.; El-Kady, M. F.; Dubin, S.; Hwang, J. Y.; Shao, Y.; Marsh, K.; McVerry, B.; Kowal, M. D.; Mousavi, M. F.; Kaner, R. B. Adv. Energy Mater. 2015, 5, 1500786. doi: 10.1002/ aenm.201500786

(30) Wu Z.; Zhang X. B. J. Electrochem. 2015, 21, 554. [吴 中, 张 新波. 电化学, 2015, 21, 554.]

(31) Yang, Z.; Ren, J.; Zhang, Z.; Chen, X.; Guan, G.; Qiu, L.; Zhang, Y.; Peng, H. Chem. Rev. 2015, 115, 5159. doi: 10.1021/ cr5006217

(32) Zou, R.; Zhang, Z.; Yu, L.; Tian, Q.; Chen, Z.; Hu, J. Chemistry 2011, 17, 13912. doi: 10.1002/chem.201101981

(33) Senthilkumar, S. T.; Selvan, R. K.; Lee, Y. S.; Melo, J. S. J. Mater. Chem. A 2013, 1, 1086. doi: 0.1039/C2TA00210H

(34) Pham, V. H.; Gebre, T.; Dickerson, J. H. Nanoscale 2015, 7, 5947. doi: 10.1039/C4NR07508K

(35) Huang, J.; Wang, J.; Wang, C.; Zhang, H.; Lu, C.; Wang, J. 
Chem. Mater. 2015, 27, 2107. doi: 10.1021/cm504618.

(36) Zheng, X.; Luo, J.; Lv, W.; Wang, D. W.; Yang, Q. H. Adv. Mater. 2015, 27, 5388. doi: 10.1002/adma.201501452

(37) Yang, J.; Yu, C.; Fan, X.; Zhao, C.; Qiu, J. Adv. Funct. Mater. 2015, 25, 2109. doi: 10.1002/adfm.201404019

(38) Wu, Z.; Huang, X. L.; Wang, Z. L.; Xu, J. J.; Wang, H. G.; Zhang, X. B. Sci. Rep. 2014, 4, 3669. doi: 10.1038/srep03669

(39) Huang, H. S.; Chang, K. H.; Suzuki, N.; Yamauchi, Y.; Hu, C. C.; Wu, K. C. W. Small 2013, 9, 2520. doi: 10.1002/ smll.201202786

(40) You, B.; Li, N.; Zhu, H.; Zhu, X.; Yang, J. ChemSusChem 2013, 6, 474. doi: 10.1002/cssc.201200709

(41) Gao, Z.; Song, N.; Li, X. J. Mater. Chem. A 2015, 3, 14833. doi: 10.1039/C5TA03057A

(42) Lu, Q.; Lattanzi, M. W.; Chen, Y.; Kou, X.; Li, W.; Fan, X.; Unruh, K. M.; Chen, J. G.; Xiao, J. Q. Angew. Chem. Int. Ed. 2011, 50, 6847. doi: 10.1002/anie.201101083
(43) Zhang, G.; Yu, L.; Hoster, H. E.; Lou, X. W. Nanoscale 2013, 5, 877. doi: 10.1039/C2NR33326K

(44) Xia, X. H.; Tu, J. P.; Wang, X. L.; Gu, C. D.; Zhao, X. B. J. Mater. Chem. 2011, 21, 671. doi: 10.1039/C0JM02784G

(45) Wang, B.; Chen, J. S.; Wang, Z.; Madhavi, S.; Lou, X. W. D. Adv. Energy Mater. 2012, 2, 1188. doi: 10.1002/aenm.201200008

(46) Brown, B.; Cordova, I. A.; Parker, C. B.; Stoner, B. R.; Glass, J. T. Chem. Mater. 2015, 27, 2430. doi: 10.1021/cm504519m

(47) Wu, Z.; Li, L.; Huang, X. L.; Zhang, X. B.; Yan, J. M. ChemNanoMat 2016, 2, 698. doi: 10.1002/cnma.201600039

(48) Zeng, Y.; Han, Y.; Zhao, Y.; Zeng, Y.; Yu, M.; Liu, Y.; Tang, H.; Tong, Y.; Lu, X. Adv. Energy Mater. 2015, 5, 1402176. doi: 10.1002/aenm.201402176

(49) Shen, L.; Che, Q.; Li, H.; Zhang, X. Adv. Funct. Mater 2014, 24, 2630. doi: 10.1002/adfm.201303138

(50) Shen, L.; Yu, L.; Wu, H. B.; Yu, X. Y.; Zhang, X.; Lou, X. W. Nat. Commun. 2015, 6, 6694. doi: 10.1038/ncomms7694 